Musées, Patrimoine et Culture scientifiques et techniques

182 | 2019

mars-avril 2019

\title{
Vers un musée humaniste et inclusif
}

\section{Nathalie Bondil, Anik Meunier et Julie Rose}

Édition électronique

DOI : 10.4000/ocim.2394

ISSN : 2108-646X

Éditeur

OCIM

Édition imprimée

Date de publication : 1 mars 2019

Pagination : $54-57$

ISSN : 0994-1908

Référence électronique

Nathalie Bondil, Anik Meunier et Julie Rose, « Vers un musée humaniste et inclusif », La Lettre de I'OCIM [En ligne], 182 | 2019, mis en ligne le 01 mars 2020, consulté le 17 mars 2020. URL : http:// journals.openedition.org/ocim/2394; DOI : https://doi.org/10.4000/ocim.2394

Ce document a été généré automatiquement le 17 mars 2020.

Tous droits réservés 


\title{
Vers un musée humaniste et inclusif
}

\author{
Nathalie Bondil, Anik Meunier et Julie Rose
}

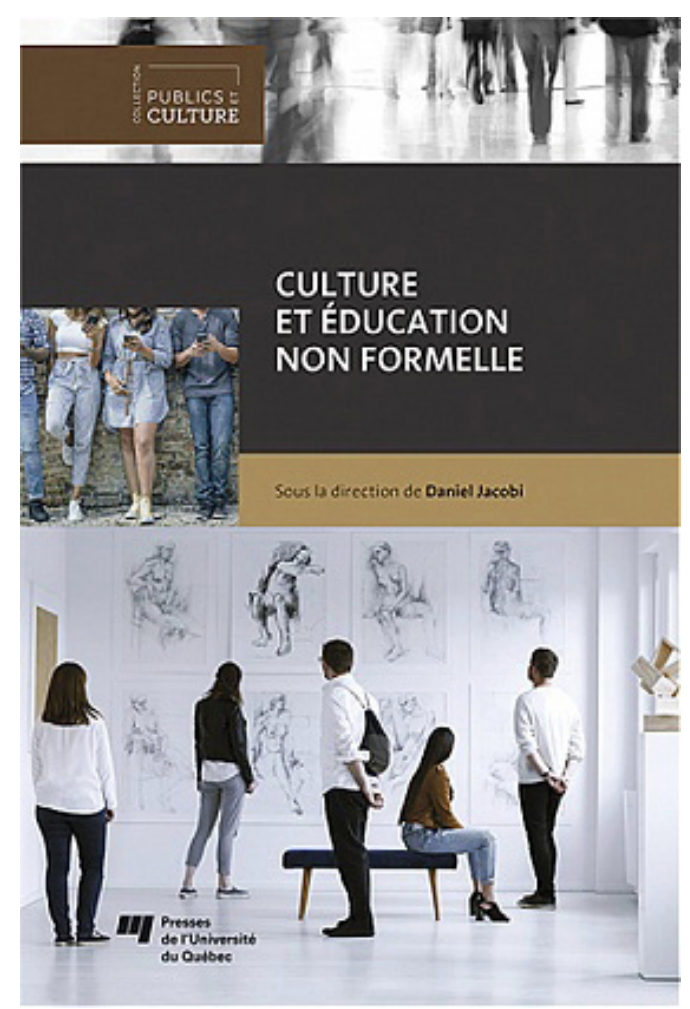

1 Julie Rose : Les musées sont des lieux de culture par excellence. Culture qui a très souvent et très longtemps été associée à des logiques savantes et expertes. Pourtant, dans une idée de démocratisation culturelle et même de démocratie culturelle, on tend à penser à un musée humaniste et inclusif, idée qui est de plus en plus répandue. On est donc en droit de se poser la question de la place de l'éducation dans cette dynamique. Ma première question va à vous, Nathalie Bondil : comment envisager l'éducation dans cette dynamique ? Le musée humaniste est-il un reflet de la société actuelle?

2 Nathalie Bondil : En fait, j'ai écrit un Manifeste pour un musée des beaux-arts humaniste dans le livre du Pavillon pour la Paix (2016) où nous nous trouvons ${ }^{1}$. Cette valeur guide 
ma vie professionnelle. Les musées sont de jeunes institutions qui ont énormément évolué et qui, probablement, continueront. Ce sont traditionnellement des lieux de collection, de conservation, de recherche, d'exposition, d'éducation et de médiation, mais je considère que la définition même du musée doit être élargie à l'inclusion... et même à la santé ! Je considère le musée comme un puissant vecteur de progrès social. Il a un rôle politique au premier sens du terme. Beaucoup de musées ont été créés comme lieux d'éducation : par exemple, le Musée des beaux-arts de Montréal, a été créé à partir de l'exemple du Victoria and Albert Museum pour montrer les meilleurs exemples d'artisanat, former les manufacturiers, éduquer le goût. Un siècle auparavant, le Victoria and Albert Museum de Londres était copié à l'échelle internationale. Aujourd'hui, naturellement d'autres missions se sont ajoutées. Or, si on apprend à lire et à écrire, on n'apprend pas à regarder. Pourtant nous sommes dans un univers visuel. Je pense que les œuvres d'art - en particulier de notre collection encyclopédique peuvent être appréhendées selon différents points de vue. C'est pourquoi j'ai imaginé Éduc'Art pour ne pas nous limiter au regard d'historien de l'art mais pour adopter une vision à 360 degrés grâce à d'autres experts : chaque œuvre est interprétée du point de vue matériel, technique, scientifique, écologique.... Les œuvres ne sont pas créées hors du monde. Au contraire, elles portent chacune un univers. Oui, les musées sont des lieux d'éducation, et l'éducation fait partie de cette mission humaniste.

3 J.R. : À un niveau beaucoup plus large, l'éducation non formelle tend donc à prendre sa place dans la société. Ma question est pour Anik Meunier : comment le musée se positionne-t-il par rapport à ce rôle?

4 Anik Meunier : Effectivement les musées sont des lieux d'éducation et s'affirment de plus en plus dans cette dynamique éducative. C'est en cela qu'on peut les considérer comme des lieux ou des espaces d'éducation non formelle. La notion d'éducation non formelle renvoie au concept de l'éducation tout au long de la vie, le « lifelong learning ». On traduit l'expression anglosaxonne de « lifelong learning " par apprentissage tout au long de la vie. Ça peut sembler un peu étrange comme façon de formuler les choses. En fait, tout observateur qui serait un petit peu attentif à la société actuelle se rend compte assez rapidement que les espaces d'éducation sont dorénavant multiformes, variés et pluriels. Le musée justement est appelé à jouer un rôle au sein de ces espaces et dans ces territoires éducatifs. Est-ce que la chose est nouvelle ? Sûrement pas. Les musées, par définition, lorsqu'ils se sont créés avaient dans leur mission des orientations éducatives. Les musées sont voués en un premier temps à préserver les collections et structurer le patrimoine comme étant le socle des valeurs symboliques d'une société. On peut d'ailleurs envisager que les musées constituent ces collections en vue de ce que l'on peut considérer comme l'identité nationale, notamment dans un souci d'instituer le patrimoine d'une collectivité. Les musées ont donc pour mission de constituer ce patrimoine à l'aide des collections, mais également de le transmettre de génération en génération. La deuxième mission des musées concerne la transmission du patrimoine et c'est assez intéressant de constater ce qui se transmet à travers le temps et ce qui est valorisé d'une façon ou d'une autre. Ces formes de valorisation et de transmission culturelle peuvent être variées et variables selon les points de vue qui sont adoptés et les époques dans lesquelles on se trouve. Et puis la troisième orientation du musée c'est celle de la délectation, le fait de créer du plaisir. Ces lieux, qui sont des lieux totalement magnifiques, des lieux de savoirs par excellence 
proposent des formes d'être. L'éducation muséale participe à cet objectif de former les visiteurs à la visite pour qu'ils soient également à l'aise dans ces lieux culturels.

N.B. : À propos d'éducation, Claude Corbo distingue le savoir-être et le savoir-faire. Je crois que le musée s'inscrit parfaitement dans cette dimension éducative qui est celle du savoir-être. Quand j'entends savoir-être, c'est aussi le savoir-être émotionnel qui, pour moi, est essentiel, une dimension que l'œuvre apporte au visiteur. Ce mode de compréhension nourrit notre intelligence sensible via l'émotion " esthétique " (soit étymologiquement des sens). On s'éduque aussi par le ressenti, et parce qu'on le connait mieux, on le maîtrise mieux. Nous parlons beaucoup d'intelligence artificielle, je m'intéresse à l'intelligence émotionnelle. Les musées sont des lieux d'éducation multiformes, je suis tout à fait d'accord.

6 A.M. : Ils s'inscrivent dans ces espaces qui ne sont plus l'école, ils ne sont pas l'école. Même si certains programmes d'études par le passé, notamment dans les années 1920, ont prôné la création de musées au sein même des établissements scolaires. C'était tout à fait louable et bien entendu, nous sommes tout à fait ravies de cela. Malheureusement, on voit qu'à travers le temps cette intention de rapprocher la culture et l'éducation, cette imbrication-là, s'est un peu perdue. Je pense que c'est aux musées aussi de redéfinir ces espaces d'éducation qui sont situés en marge du cadre scolaire. Mon propos n'est pas ici de critiquer l'école. C'est l'outil par excellence pour former aux différentes sources de culture et de savoirs. Mais il faut rappeler que les formes d'appropriation de ces savoirs culturels sont aussi mises en œuvre à l'extérieur de l'école.

7 N.B. : J'étais enchantée de lire dans La Presse un portrait de la nouvelle ministre de la Culture du Québec, Nathalie Roy, dont l'une des premières décisions, est d'accorder deux sorties culturelles aux écoles. Bravo ! Beau geste comme première décision ministérielle ! Cependant, gardons-nous de considérer la visite au musée comme un " divertissement ». Le musée est sorti de l'orbite éducative ce qui n'était pas le cas il n'y a pas si longtemps. D'un point de vue étymologique, «se divertir » signifie « se détourner de soi ", c'est donc participer à un courant plus mainstream alors qu'en fait les musées sont des lieux d'éducation et de recentrement, d'émotion et de réflexion en même temps: ce n'est pas tant que les musées se soient éloignés de l'éducation qu'une certaine vision qui les en a éloignés davantage axée sur l'évènementiel. Donc c'est à nous, professionnels de musée, de rendre accessibles nos collections via les contenus scolaires, de les externaliser jusqu'aux bancs d'école et dans les classes via nos plateformes numériques et nos objets nomades. C'est à nous d'écouter les besoins des professeurs et de nous adapter aux cursus d'enseignement pour co-créer des contenus pertinents qui seront utiles... et utilisés. C'est pourquoi, pour ÉducArt, j'ai voulu embaucher des professeurs pour travailler avec leurs collègues. Par exemple, un cours sur Louis XIV évoquera aussi bien les Filles du Roi en Nouvelle France que le rare modello du célèbre portrait royal peint par Rigault que nous avons dans notre collection, qui appartient à notre patrimoine collectif. Pour les enseignants comme pour leurs élèves, il sera aussi évident d'évoquer le Roi Soleil via un manuel scolaire qu'une œuvre d'art. L'apprentissage et l'éducation ne se font pas simplement par le biais du mot mais aussi par le son, la vision : il existe bien d'autres langages. Cette multiplicité de canaux du savoir nous éduque. Culture et éducation sont des sœurs siamoises. 
8 A.M. : Cette dimension est toutefois absente de la formation des maîtres. Il faut quand même le rappeler parce qu'au-delà de ces intentions, tout à fait nobles, il y a une praxéologie qui doit être mise en œuvre. Malheureusement, cette pratique professionnelle, cette pratique enseignante ne va pas nécessairement avoir recours à ces formes d'art, que ce soit le musée des beaux-arts, que soit les musées d'histoire, les musées de science et technologie également. Il y a cette nécessité de rapprocher les deux univers. Oui, culture et éducation sont des sœurs siamoises, mais je pense qu'elles ont été un peu dissociées. L'aspect qui est fort interpelant dans toute cette dynamique c'est que ceux qui vont être auprès des jeunes, les enseignants, n'ont souvent, euxmêmes, pas développé cette pratique de la fréquentation des lieux culturels. Cette pratique culturelle ne se trouve pas non plus dans leur formation initiale. Il n'y a malheureusement aucun incitatif, ni aucune mesure enjoignant les enseignants à développer cette habitude de fréquentation des lieux culturels ou des musées, ni même leur utilisation avec leur classe. À la limite, si le musée était instrumentalisé, au pire, il serait instrumentalisé et les enseignants viendraient avec leurs élèves fréquenter les musées, voir des œuvres d'art. Je m'explique en fait parce que...

$9 \quad$ N.B. : Pourquoi instrumentalisé ? J'ai l'impression que quand on entre dans un musée on doit justement ne pas instrumentaliser en raison d'une espèce de noblesse et d'un droit de la parole que l'on ne s'accorde pas. Pour moi, le droit de parole dans un musée n'appartient pas seulement aux conservateurs, experts et historiens d'art. Il appartient à tout le monde. L'art est polysémique, c'est sa force. Toutes sortes d'experts ont la capacité de pouvoir s'exprimer pour interpréter les œuvres avec pertinence et pour échafauder leur savoir. C'est très important de casser ce monopole de l'interprétation qui nous cadenasse. Plusieurs cartes peuvent lire le territoire du musée et de ses collections : quelle chance!

10 A.M. : Absolument, mais l'interprétation est encore tellement présente. Je ne serais pas si triste non plus, bien au contraire, si mes propos étaient les suivants : le fait d'instrumentaliser le musée n'est pas un souci en soi. C'est peut-être même je dirais la première étape dans l'appropriation véritable du musée. Alors le fait de pouvoir établir des liens avec les contenus scolaires et culturels serait le degré zéro de l'approche de l'école au musée, ou des enseignants avec leurs élèves. Mais on n'en est même pas là ! On n'en est même pas à l'instrumentalisation du musée pour des fins éducatives. Alors on peut imaginer qu'au-delà de la démocratisation, on est bien loin de ça.

11 N.B. : Néanmoins, je pense qu'il faut séparer le contenant du contenu, la scène de son répertoire ou le musée de ses collections.

A.M. : Absolument.

N.B. : Que ce soit en musique classique, en théâtre ou en arts visuels, ce contenu doit être apporté beaucoup plus tôt dans l'éducation. Et en fait, il existe peu de pays qui intègrent l'histoire des arts au cursus scolaire. Par exemple en Italie, les cours comprennent une histoire des arts. Les jeunes Italiens sont sensibilisés à leur patrimoine artistique et à leur passé culturel remarquables. En France, Pierre Rosenberg, ancien directeur du Louvre, aujourd'hui à l'Académie française, a poussé l'enseignement de la culture et des arts à même l'éducation, malheureusement sans professeurs spécialisés d'où des mécontentements et une surcharge pédagogique. La difficulté que j'ai rencontrée en venant au Québec, c'est qu'il y a non seulement, peu de place faite à la culture dans les cours mais tout simplement à l'histoire. De plus, une population d'origine très diversifiée ici en métropole oblige à repenser les narrations, 
moins ethnocentrées et plus globalisées. C'est donc plus compliqué de se rattacher à un enseignement sans notions basiques d'histoire collective. Pourtant notre Pavillon d'art québécois et canadien, c'est une leçon d'histoire. C'est un manuel que l'on visite avec ses pieds au lieu de le parcourir avec ses yeux. C'est exactement la même chose. Mais encore faut-il qu'il y ait cette volonté dans le cadre même des cursus scolaires de renforcer cette dimension, au cœur des enjeux interculturels et des questions actuelles. Puisque l'histoire est si peu présente en classe, j'ai voulu trouver d'autres stratégies pour amener nos collections, incluant les cultures du monde, dans le quotidien des élèves. En tant que conservatrice amoureuse de nos collections, ma mission est qu'elles vivent au travers d'autres regards, d'autres questionnements qui les rendent pertinentes et intéressantes. J'ai donc voulu explorer les sciences de la vie, sociales ou humaines... ce qui a donné naissance à notre ambitieuse plateforme pédagogique, Éduc'Art. Ce projet transdisciplinaire analyse nos collections par d'autres voies que celles traditionnellement liées à la culture... et ça fonctionne. On est en train de l'étudier, n'est-ce pas?

14 A.M. : Éduc'Art est un magnifique projet pour permettre que le musée vienne vers les écoles ou dans les écoles et ça c'est tout à fait remarquable. Surtout parce qu'il se déploie dans un univers qui n'est pas géographique. C'est un des avantages en tout cas. Mais est-ce que ça ne témoigne pas aussi de la difficulté de faire venir les enseignants dans le musée avec leurs élèves ? Concrètement sur les lieux physiques et parcourir ce manuel scolaire à pied. Dans ce contexte-là peut-être qu'on aurait réfléchi un projet comme Éduc'Art de manière différente et c'est pour ça que je me dis qu'à mon sens les bases entre la culture et l'éducation ne sont encore même pas établies. Elles ne sont pas encore articulées ou arrimées. Donc la formation initiale des maîtres, pour en revenir là, me semble tout à fait le créneau ou à tout le moins une des voies à explorer pour faire bénéficier les jeunes de la culture dont recèlent les musées. Certainement pour que cette articulation entre l'éducation et les savoirs du musée puisse justement imprégner les savoirs de l'école.

J.R. : Je n'ai rien eu à faire. On m'a demandé une animation.

N.B. : Quel est ton avis, en tant qu'étudiante?

A.M. : Oui ça serait intéressant. Julie s'intéresse à quelque chose de fascinant en muséologie. Elle va nous en parler un peu.

18 J.R. : La fréquentation, la non-fréquentation des adolescents au musée.

N.B. : Qu'est-ce que tu proposerais alors pour amener plus d'adolescents au musée ?

J.R. : C'est un peu difficile de se positionner, comme ça, maintenant. J'avoue que je ne sais pas encore, puisque je viens de terminer ma collecte de données. Donc j'attends de pouvoir l'analyser pour en sortir un peu plus d'informations, mais il est clair que le musée reste vital et présent dans la vie des adolescents puisqu'ils sont contents d'y aller même si c'est à reculons dans certains cas. Notamment, avec l'école, mais ça reste ouvert à la discussion. À voir plutôt début 2019, quand j'aurai terminé.

21 A.M. : Mais tu peux quand même nous mettre un peu en appétit, parce que tu es allée rencontrer des adolescents. Qu'est-ce qu'ils t'ont dit ? Il y a les plus jeunes adolescents, mais il y a ceux qui commencent à avoir leur indépendance, qui deviennent de plus en plus autonomes. Il y a deux tranches d'âge que tu as observées, c'est ça ?

J.R. : Ceux qui ont moins de 14 ans sont très heureux d'y venir en famille et sont super contents d'y aller, c'est une belle activité. Passé 14 ans, c'est un peu spécial. D'un coup 
ça devient une activité où on traîne les pieds, où on suit le petit frère, la petite sœur parce que soudainement ce sont eux qui ont très envie d'aller au musée. Et les adolescents ont autre chose à faire dans la vie. Quoi ? On ne sait pas, mais ils ont autre chose à faire. Les plus vieux ont l'impression d'être plus ou moins compris par le musée. Ils se demandent si on les prend encore pour des gamins. J'ai notamment une jeune dans un des groupes qui m'a dit : «Est-ce que les musées nous font confiance? " Voilà, donc je laisse planer ça et puis on verra plus tard.

\section{NOTES}

1. Bondil, N. Manifeste pour un musée des beaux-arts humaniste. Musée des beaux-arts de Montréal, Pavillon pour la paix Michal et Renata Hornstein, Artinternational et éducation, Atelier international d'éducation et d'art thérapie Michel de la Chenelière, Montréal, 2016, pp. 20-28.

\section{RÉSUMÉS}

Cet entretien entre Nathalie Bondil, directrice générale et conservatrice en chef du Musée des beaux-arts de Montréal et Anik Meunier, professeure titulaire en éducation et muséologie et directrice du Groupe de recherche sur l'éducation et les musées (GREM) de l'Université du Québec à Montréal (UQAM), a eu lieu le $1^{\mathrm{er}}$ novembre 2018 à l'occasion du lancement du livre Culture et éducation non formelle. Cet ouvrage collectif dirigé par Daniel Jacobi est le dixième titre de onze publiés dans la collection "Culture et publics ", dirigée par Anik Meunier et Jason Luckerhoff, professeur titulaire en culture et communications de l'Université du Québec à Trois-Rivières (UQTR). L'animation a été assurée par Julie Rose, étudiante à la maîtrise en muséologie et assistante de recherche au sein du GREM de l'UQAM.

\section{INDEX}

Mots-clés : Québec, éducation non-formelle, publics

\section{AUTEURS}

\section{NATHALIE BONDIL}

Nathalie Bondil, directrice générale et conservatrice en chef du Musée des beaux-arts de Montréal. 


\section{ANIK MEUNIER}

Anik Meunier est professeure titulaire en éducation et muséologie et directrice du Groupe de recherche sur l'éducation et les musées (GREM) de l'Université du Québec à Montréal (UQAM).

\section{JULIE ROSE}

Julie Rose est étudiante à la maîtrise en muséologie et assistante de recherche au sein du GREM de l'UQAM 